\title{
The use of augmented reality glasses in central line simulation:"see one, simulate many, do one competently, and teach everyone"
}

This article was published in the following Dove Press journal:

Advances in Medical Education and Practice

\section{Cynthia Y Huang' Jonathan B Thomas ${ }^{2}$ \\ Abdullah Alismail ${ }^{3}$ \\ Avi Cohen' \\ Waleed Almutairi ${ }^{3}$ \\ Noha S Daher ${ }^{4}$ \\ Michael H Terry ${ }^{5}$ \\ Laren D Tan',3}

'Department of Medicine, Division of Pulmonary, Critical Care, Hyperbaric and Sleep Medicine, Loma Linda University, Loma Linda, CA, USA; ${ }^{2}$ Zapara School of Business, La Sierra University, Riverside, CA, USA; ${ }^{3}$ Cardiopulmonary Sciences Department, School of Allied Health Professions, Loma Linda University, Loma Linda, CA, USA; ${ }^{4}$ Department of Allied Health Studies, School of Allied Health Professions, Loma Linda University, Loma Linda, CA, USA; ${ }^{5}$ Department of Respiratory Care, Loma Linda University Medical Center, Loma Linda, CA, USA
Correspondence: Laren D Tan Department of Medicine, Division of Pulmonary, Critical Care, Hyperbaric and Sleep Medicine, Loma Linda University School of Medicine, I 234 Anderson Street, Suite 6439, Loma Linda, CA 92354, USA

Tel + I 909558808 I

Fax + I 909558 058I

Email LaTan@llu.edu
Objective: The aim of this study was to investigate the feasibility of using augmented reality (AR) glasses in central line simulation by novice operators and compare its efficacy to standard central line simulation/teaching.

Design: This was a prospective randomized controlled study enrolling 32 novice operators. Subjects were randomized on a 1:1 basis to either simulation using the augmented virtual reality glasses or simulation using conventional instruction.

Setting: The study was conducted in tertiary-care urban teaching hospital.

Subjects: A total of 32 adult novice central line operators with no visual or auditory impairments were enrolled. Medical doctors, respiratory therapists, and sleep technicians were recruited from the medical field.

Measurements and main results: The mean time for AR placement in the AR group was $71 \pm 43 \mathrm{~s}$, and the time to internal jugular (IJ) cannulation was $316 \pm 112 \mathrm{~s}$. There was no significant difference in median (minimum, maximum) time (seconds) to IJ cannulation for those who were in the AR group and those who were not $(339$ [130, 550] vs 287 [35, 475], $p=0.09)$, respectively. There was also no significant difference between the two groups in median total procedure time $(524[329,792]$ vs $469[198,781], p=0.29)$, respectively. There was a significant difference in the adherence level between the two groups favoring the AR group $(p=0.003)$.

Conclusion: AR simulation of central venous catheters in manikins is feasible and efficacious in novice operators as an educational tool. Future studies are recommended in this area as it is a promising area of medical education.

Keywords: augmented reality, central line, simulation, medical education

\section{Introduction}

A central venous catheter (CVC) is frequently used with more than 5 million that are sold each year in the USA alone ${ }^{1}$ and is used for hemodynamic monitoring as well as the administration of fluids and medications that cannot be otherwise administered through a peripheral vein. Education regarding the use of intravascular devices and proper insertion technique is recommended by the Center for Disease Control and Prevention (CDC) and Healthcare Infection Control Practices Advisory Committee guidelines. ${ }^{2}$ Despite the integration of bedside ultrasound into the standard of practice, ${ }^{3}$ the immediate complication rates still range from $4 \%$ to $7 \%{ }^{4}$ with up to a $12 \%$ complication rate in inexperienced operators (compared to $0 \%$ in experienced operators). ${ }^{5}$ The classic teaching model in CVC insertion is "see one, do one, teach one," ${ }^{6-8}$ but learning such complex skills, which themselves have risks to the patients, for the first time in the complex work environment where there are many distractions 
and competing factors is suboptimal. ${ }^{9}$ It is an ethical dilemma and spotlights the balance of risk to the patient while providing education for the novice medical professional; ${ }^{10}$ prior studies from the Institute of Medicine have shown that deaths due to medical errors exceed death from motor vehicle accidents. ${ }^{11}$

Simulation-based learning has been shown to reduce complications ${ }^{12,13}$ and is now an integral part of medical education. It allows for teaching of complex skills in a controlled environment, one away from the judgment or anxiety of dealing with a real patient, it affords learners the ability to make errors without any real-life adverse consequences, and it provides instructors who are focused completely on their education. ${ }^{9}$ Specific to CVC insertion, simulation has been shown to improve procedural knowledge, adherence with a standard protocol checklist, and safety elements (and thus decreasing the rate of catheter-related bloodstream infections from 3.20 per 1,000 catheter days to 0.50 per 1,000 catheter days $\left.{ }^{14}\right)$. However, subjects still fail to wash their hands, prepare with chlorhexidine, drape the patient using sterile technique, anesthetize the site, and perform a preprocedure time-out. ${ }^{15}$ These items have been proven to be integral to procedural safety and prevention of catheterrelated bloodstream infections. ${ }^{2}$ Government organizations, such as Medicare, no longer reimburse for preventable central line placement complications. ${ }^{16}$ Thus, the minimization of complications is essential — both for the patient and for the utilization of resources.

Augmented reality (AR) is defined as "technology" that merges the real- and virtual-world experience. ${ }^{17,18} \mathrm{AR}$ is one of the key emerging technologies in education ${ }^{19,20}$ and has already been studied in teaching surgical techniques. ${ }^{21-24}$ The use of AR to augment current CVC simulation-based teaching would provide standardized real-time instruction as well as increase compliance with the recommended bundle. By using a virtual interface to provide a 1) review checklist (Table 1, data from the Joint Commission ${ }^{25}$ ) and 2) real-time instruction, it can better ensure that the novice user has the necessary equipment and is learning as well as performing standard of practice care (quality indicators, ie, sterile field). Thus, the goal with the advent of AR technology is for medical trainees to "see one, simulate many, do one competently, and teach everyone."

The purpose of this study was to investigate the feasibility and effectiveness of the use of AR glasses on CVC placement in selected health-care professionals. The study outcome measures were time of procedure completion and the subject's feedback on the future use of AR in CVC. We hypothesized
Table I Checklist

Sterile field maintained

Each lumen of catheter flushed with saline prior to placement

Catheter caps placed on all lumens

All lumens clamped

Confirmation of venous placement of guidewire prior to dilatation of vein by ultrasound

Blood aspirated from each lumen

Catheter secured

Sterile dressing applied (gauze, transparent dressing, gauze and

transparent dressing, antimicrobial foam disk)

Confirmation of catheter placement by chest X-ray

Qualified second operator obtained after three unsuccessful sticks

Note: Data from the Joint Commission. ${ }^{25}$

that the AR simulation-based CVC insertion training will decrease complication rates and increase compliance with quality indicator measures by providing real-time feedback as well as unifying all the important information onto one screen for the provider.

\section{Subjects and methods Protocol}

A total of 32 subjects were recruited and randomized into two groups: 1) traditional simulation and 2) AR simulation (Figure 1). An excel randomization table was used for the randomization process. All subjects filled out a demographic questionnaire regarding their background and their perceptions of AR glasses in CVC and the use of AR glasses in medical education. All groups watched an instructional video prior to simulation (ultrasound-guided internal jugular [IJ] vein cannulation). ${ }^{26}$ At the end of the instructional video, subjects were divided into their designated groups (Figure 1).

\section{Control group}

Traditional simulation consisted of a subject using the ultrasound to attempt an IJ vein central line insertion on a manikin. A designated observer served as the grader, recording the start time, and completion of the checklist (Figure 2). If needed, the observer was also allowed to answer questions and provide real-time feedback (with that documented on the data collection form).

\section{AR group}

The AR simulation group had a 5-10-min hands-on instructional course to allow familiarity with the AR equipment. They then proceeded to attempt an IJ vein insertion on the same manikin model as the control group. As in the other arm, a designated observer served as the grader, recording the start time, and completion of the checklist. 


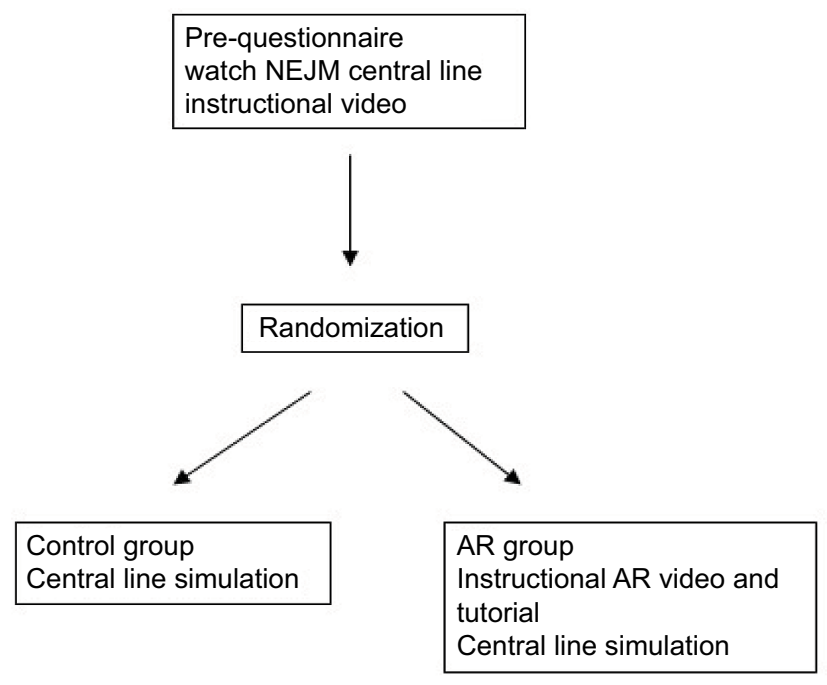

Figure I Schematic of study protocol.

Abbreviations: AR, augmented reality; NEJM, the New England Journal of Medicine.

\section{AR glasses and setup}

The Brother AiRScouter WD-200B AR glasses (Brother International Corp., Bridgewater, NJ, USA) comes with one display unit, with a headband and a control box (Figure 3). Prior to using the AR glasses, a tutorial was provided by a test administrator on the basics of the AR glasses. Subjects were instructed to position the eyepiece slightly to the side of the eye, as to not cover the entire eye and inhibit bifocal vision. They were also given the opportunity to adjust the display in the eyepiece by moving the eyepiece right or left, toward or away from the eye. If only half the image was visible, subjects were instructed to move the eyepiece by moving it either toward or away from the eye, while holding the arm steady in one place.

Displayed on the eyepiece was a concise CVC instructional slide show adapted from the CVC instructional video on the New England Journal of Medicine ${ }^{26}$ (simulated views are shown in Figure 4). The slides were used as a checklist for subjects to follow as they carried out the procedure. Each slide included only key instructional words from the procedure manual. Microsoft PowerPoint 2016 on a laptop computer (connected to the AR glasses) was used to present the instruction slides. A wireless two-button foot pedal was connected to the computer via Bluetooth to advance the slides. When the pedal on the right was pressed, the slides would advance. When the pedal on the left was pressed, the slide would go back. The PowerPoint Slides displayed on the AR glass used 72 font green colored text that was on top of a black background to increase user clarity and reduce eyestrain. On some slides, photos and short gifs were displayed (ie, the image of a vein or an artery).

\section{The Use of Augmented Reality in Central Line Simulation Data Collection Sheet}

Date:

Age:

Sex: Male/Female

Subject \#: Investigator Name:

Enrollment: [ ] AirScouter with Training

[ ] No intervention

Time AR set on Head:

Time to IJ Cannulation:

Total Time:

\# of Connulation Attempts:

\# of Artery Sticks:

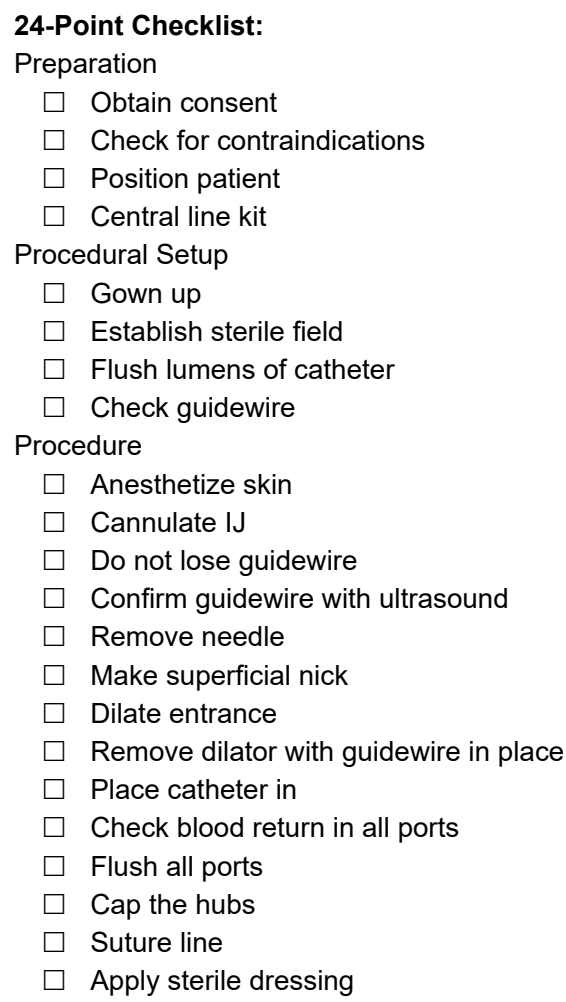

Figure 2 Data collection sheet.

Abbreviations: AR, augmented reality; IJ, internal jugular.

\section{Bias prevention}

During all simulations, only the study subjects actively engaged in the central line simulation were present in the simulation area. This was performed to control for bias in repeated studies (eg, the second person watching the first would have an advantage).

\section{Data collection}

The designated observer recorded and documented compliance with the performance criteria utilizing a checklist recording the number of attempts, the time, and the success/ 
failure of each attempt (Figure 2). At the end of their experience, each subject completed a questionnaire to record their overall satisfaction with the simulation experience and their comfort and proficiency at the end of the process. The survey's assessment of the AR glasses was based on the questionnaire developed and validated by Santos et $\mathrm{al}^{27}$ at Nara Institute of Science and Technology.

\section{Statistical analyses}

A sample size of 32 subjects was estimated using a large effect size of 0.9 , a power of 0.80 , and the level of significance set at 0.05 . Data were summarized using mean $\pm \mathrm{SD}$ or median (minimum, maximum) for quantitative variables and frequency

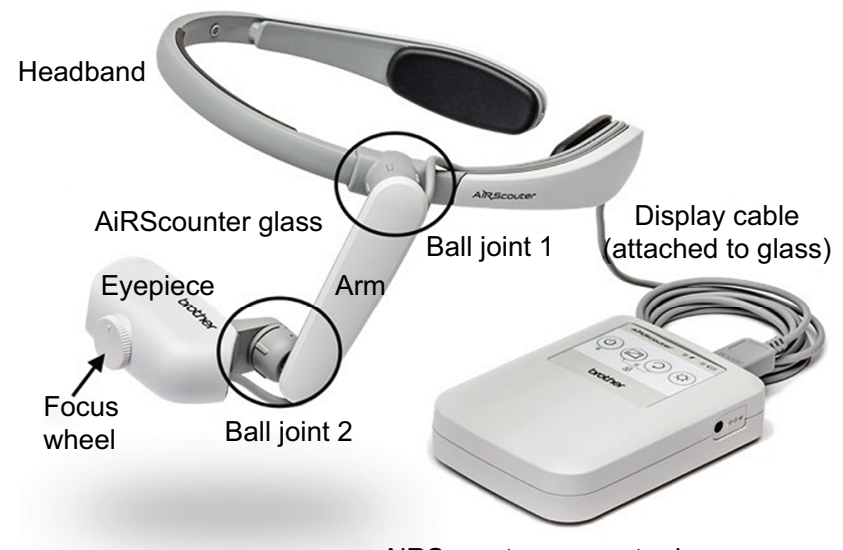

AiRScounter connector box

Figure 3 AiRScouter WD-200B.

Note: Not shown in this figure is the head strap that connect from one rear part of the headband to the other.

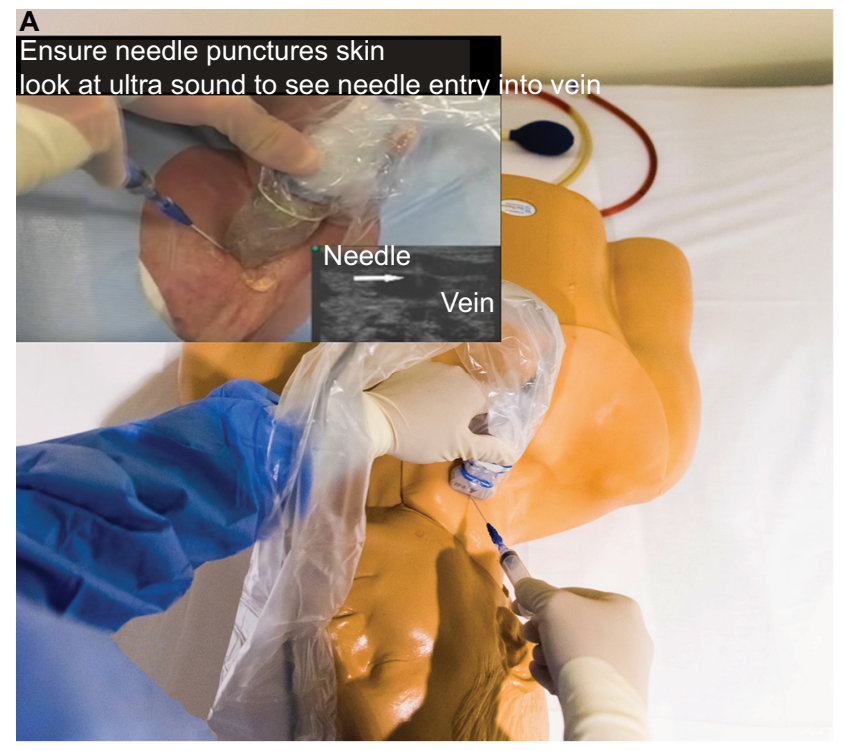

(\%) for categorical variables. The normality of the quantitative outcomes was assessed using Kolmogorov-Smirnov test. The distribution of categorical characteristics of subjects by the study group was examined using Pearson's chi-square test. An independent $t$-test was used to compare mean age between the two study groups. Mann-Whitney test was used to compare time to IJ cannulation and adherence between the two study groups. The level of significance was set at $p \leq 0.05$. Thematic analysis was used to analyze subjects' comments regarding the use of $A R$ in the future for the AR group.

\section{Ethical committee approval and recruitment}

The study was approved by the institutional review board of Loma Linda University Health, Loma Linda, CA, USA. Subject recruitment was performed by distributing flyers and emails in our health-care institution. All subjects signed an informed consent upon recruitment to the study.

\section{Results}

A total of 32 subjects participated in this study (respiratory therapists, $n=19$ [59\%]; physicians, $n=11$ [34\%]; sleep technicians $n=2[6 \%])$. The mean age for all subjects was $29.8 \pm 7.8$ years, with the majority being males $(n=27,71 \%)$. There was no significant difference in baseline characteristics between the two groups ( $p>0.05$; Table 2$)$. For baseline questionnaires, most subjects agreed that AR would be easy to use when placing central lines, would decrease procedure time, and would decrease the number of required attempts,

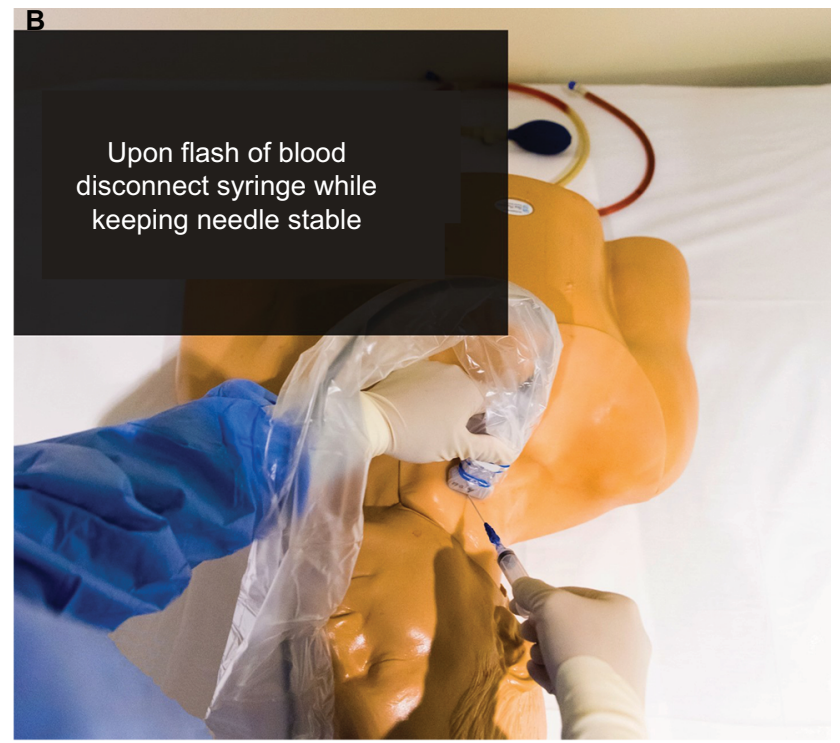

Figure 4 Simulated view through AiRScouter glasses.

Notes: (A) Image displayed through glasses gives instruction of what to look for when placing the needle in the vein. (B) Text displayed through glasses gives instruction for the next step of the procedure. 


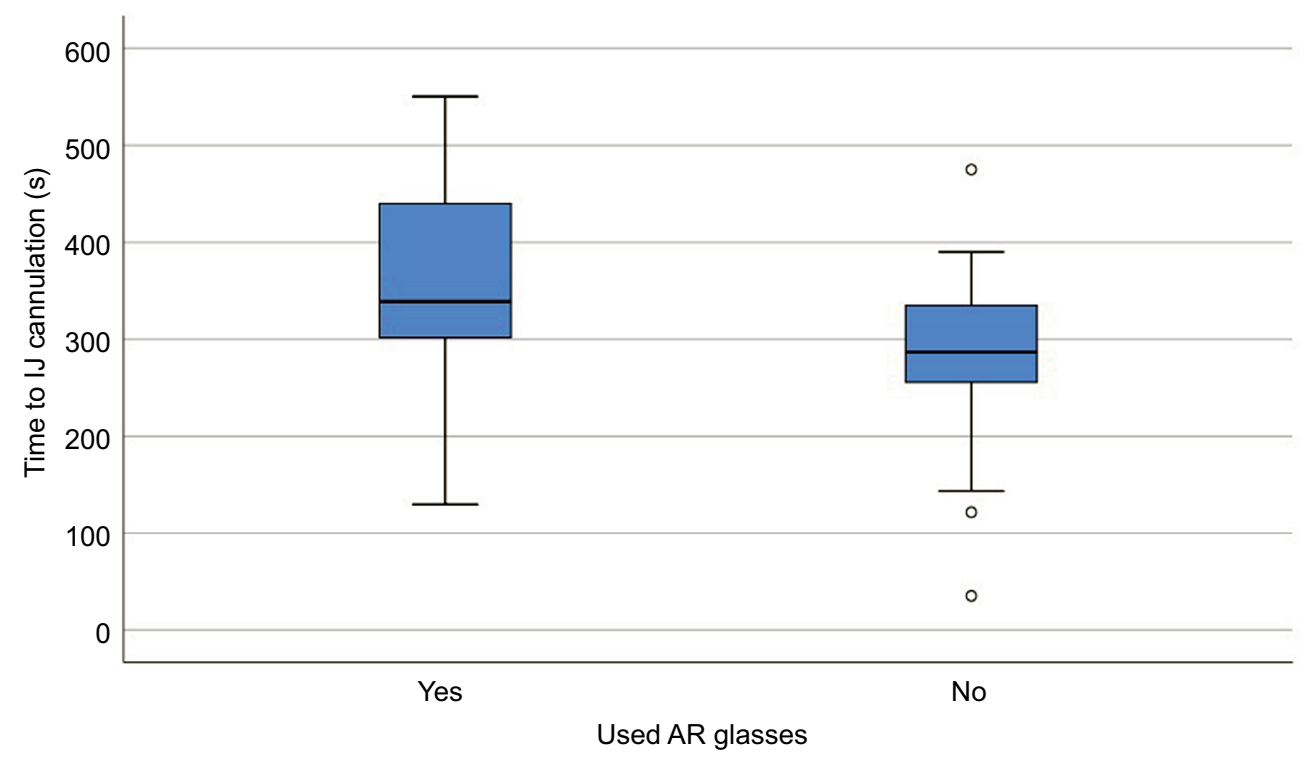

Figure 5 Time to IJ cannulation by $A R$ usage $(\mathrm{N}=32)$.

Abbreviations: AR, augmented reality; IJ, internal jugular.

Table 2 Frequency distribution of characteristics of subjects by the use of $A R$ glasses $(\mathrm{N}=32)$

\begin{tabular}{lll}
\hline Characteristics & $\begin{array}{l}\text { AR } \\
\left(\mathbf{n}_{\mathbf{1}}=17\right)\end{array}$ & $\begin{array}{l}\text { Non-AR } \\
\left(\mathbf{n}_{\mathbf{2}}=1 \mathbf{1 5}\right)\end{array}$ \\
\hline Male & $13(76.5 \%)$ & $9(60 \%)$ \\
Age (mean $\pm S D)$ & $27.5 \pm 3.1$ & $32.4 \pm 10.6$ \\
$\begin{array}{l}\text { Prior experience with CVC in human beings } \\
\text { Experience central lines with manikins }\end{array}$ & $4(23.5 \%)$ & $6(40 \%)$ \\
$\begin{array}{l}\text { Profession } \\
\quad \text { Respiratory therapy }\end{array}$ & $5(33.3 \%)$ \\
$\quad \begin{array}{l}\text { Medical doctor } \\
\text { Sleep technologist }\end{array}$ & $9(52.9 \%)$ & $10(66.7 \%)$ \\
\hline
\end{tabular}

Abbreviations: AR, augmented reality; CVC, central venous catheter.

and nearly all agreed that AR would be helpful in medical education curriculums (in respective order $n=24$ or $80 \%$; $\mathrm{n}=21$ or $66 \% ; \mathrm{n}=23$ or $72 \% ; \mathrm{n}=29$ or $91 \%$ ). Subjects were split regarding whether $\mathrm{AR}$ would require much training and whether it would have significant side effects to the user (50\% and 53\%).

The distribution of time to place the AR device on head and time for central lines and adherence were not proportionally equal. The mean time for AR placement on the head in the AR group was $71 \pm 43 \mathrm{~s}$, and the time to IJ cannulation was $316 \pm 112 \mathrm{~s}$. The mean total time of completion for all subjects was $483 \pm 144 \mathrm{~s}$. There was no significant difference in median (minimum, maximum) time (seconds) to IJ cannulation for those who were in the AR group and those who were not (339 [130, 550] vs 287 [35, 475], $p=0.09$; Figure 5), respectively. There was also no significant difference between the two groups in median total procedure time (524 [329, 792] vs 469 [198, 781], $p=0.29)$, respectively. The majority of the subjects $(71 \%, n=23)$, overall, were successful in cannulation of the IJ on their first attempt (12 in the AR group vs 11 in the non-AR group). Chi-square test showed no significant difference between the two groups when it comes to cannulation attempts. To test the hypothesis that the AR group performed better than the non-AR group in the adherence checklist, Mann-Whitney test was performed. Results showed that there was a significant difference in adherence level between the two groups $(22.9 \pm 4.1$ in the AR group vs $18.1 \pm 6.3$ in the non-AR group; $\eta^{2}=0.90, p=0.003$ ).

In the postquestionnaire for the AR group, the majority ( $>80 \%$ ) reported that the device did not cause any fatigue, is not bulky, or too heavy after use in terms of ergonomics. Similarly, 94\% reported that hand, head, and foot interactions were not difficult to perform and did not require additional work. With regard to comprehensibility, about $80 \%$ believed that the information displayed on the screen was appropriate, not difficult to read, and was responding fast enough. Similarly, for manipulability, the majority believed that the device did not require a lot of body muscle effort, and it was comfortable for arms and hands. However, $30 \%$ believed that the device interfered with their procedure skills and that it was not easy to control.

Thematic analysis for the comments in the AR group, showed mutual agreement of the usefulness of the device in the medical field overall. However, issues with device adjustments during the procedure were one of the main 
complaints subjects had. In addition, most subjects commented that the device required more training and practice than was allocated in this study before they would feel comfortable with it to perform the procedure.

\section{Discussion}

The Institute of Medicine report published in $1999^{11}$ brought to light the importance for developing systems and processes to improve patient safety in hospitals. While there has been an emphasis on developing systems to prevent CVC-associated complications via Center for Disease Control and Prevention checklists and Centers for Medicare \& Medicaid Services tracking of performance measures, ${ }^{28}$ there is still much room to improve the complication rates in novice (vs expert) operators in which complication rates were $11 \%$ and failure rates $19.4 \%$ in one study (vs $4.5 \%$ and $10.1 \%$, respectively, in expert operators). ${ }^{29}$ The ethical dilemma of balancing medical education with patient safety has always been a fundamental issue in medical training. In addition, the medical reforms with the decreases in training hours brought on by the Accreditation Council for Graduate Medical Education ${ }^{30,31}$ have further challenged the system to condense the same amount of information to learn into fewer working hours. Simulation has been extensively studied, ${ }^{10,12,15,24,32-34}$ but there is still a gap between simulation and performing actual procedures. In our study, we evaluated the use of AR to bridge the chasm, by providing real-time instruction during simulation training with future plans to study its implementation in the real-life environment.

Since central lines are an integral part in patient care, with up to 5 million annually placed in the USA alone, ${ }^{35}$ it is an important issue that must be explored. AR has already been proven to be a powerful teaching tool in various nonmedical fields (ie, aviation, business, and military), and its use has been explored in the training of emergency room physicians and surgeons..$^{7,33,34}$ Our study is the first, to our knowledge, to explore the use of AR glasses in central line simulation. We have shown that AR can be used during simulation CVC training without significant limitations to the user. Our results showed that there was a significant difference in the adherence level in the AR group vs non-AR group. This significant increase can be explained by the real-time feedback the AR group received as they perform the procedure.

Prior studies have identified that AR brings its own cohort of unique problems. ${ }^{36}$ For example, students reported feeling overwhelmed and confused when dealing with unfamiliar technologies in the setting of complex tasks. ${ }^{37}$ However, our study shows that study subjects only required a mean of $71 \pm 43 \mathrm{~s}$ to implement the AR glasses and that despite the time required to place the glasses on, there was no significant difference in time to IJ cannulation. In addition, our postquestionnaire for the AR group reveals that the AR glasses were easy to use and did not require a lot of work to operate. Although some subjects faced some challenges in using the AR glasses (30\%), this can be seen in other reported studies as well. Akçayır and Akçayır ${ }^{38}$ reported, in their systematic review, that the most challenging aspect of AR is usability issues and technical problems. We thus recommend that future studies examine the usability and easiness of device usage during a procedure. In addition, based on the subjects' comments, we believe that more training needs to be provided to the user in the future studies.

\section{Limitations}

Having a larger number of subjects in the future studies would show better promising results of the use of AR. In addition, we believe that future studies should assess AR's effect longitudinally - after its use in simulation - and to assess the efficacy of naive operators' placements of central lines in live patients. This will give a better measurement and assessment of the AR effectiveness as a medical education tool. Finally, future studies are also warranted to investigate safety and reliability of AR during placement of central line catheters in live patients. In addition, future studies should consider previous experience on the procedure as an important factor in assessing the usefulness of AR.

\section{Conclusion}

AR simulation of CVCs in manikins is feasible and efficacious in novice operators as an educational tool. Future studies in this area are encouraged as technology is advancing rapidly in this area of education.

\section{Acknowledgments}

We are indebted to Brother International Corp., Bridgewater, NJ, USA, Tom \& Vi Zapara School of Business at La Sierra University and Loma Linda University Ignis Medical Innovations (IMI) for their their support during this study.

\section{Disclosure}

The authors report no conflicts of interest in this work.

\section{References}

1. Maki DG. Infections caused by intravascular devices used for infusion therapy: pathogenesis, prevention, and management of infections due to intravascular therapy. In: Bisno AL, Waldvogel FA, editors. Infections Associated with Indwelling Medical Devices. 2nd ed. Washington, DC: American Society for Microbiology; 1994:155-212. 
2. Safdar N, Kluger DM, Maki DG. A review of risk factors for catheterrelated bloodstream infection caused by percutaneously inserted, noncuffed central venous catheters: implications for preventive strategies. Medicine. 2002;81(6):466-479.

3. Randolph AG, Cook DJ, Gonzales CA, Pribble CG. Ultrasound guidance for placement of central venous catheters: a meta-analysis of the literature. Crit Care Med. 1996;24(12):2053-2058.

4. Kornbau C, Lee KC, Hughes GD, Firstenberg MS. Central line complications. Int J Crit Illn Inj Sci. 2015;5(3):170-178.

5. Lefrant JY, Muller L, De La Coussaye JE, et al. Risk factors of failure and immediate complication of subclavian vein catheterization in critically ill patients. Intensive Care Med. 2002;28(8):1036-1041.

6. Rodriguez-Paz J, Kennedy M, Salas E, et al. Beyond "see one, do one, teach one": toward a different training paradigm. Qual Saf Health Care. 2009;18(1):63-68.

7. Vozenilek J, Huff JS, Reznek M, Gordon JA. See one, do one, teach one: advanced technology in medical education. Acad Emerg Med. 2004;11(11):1149-1154.

8. Tuthill J. See one, do one, teach one. Lancet. 2008;371(9628):1906.

9. Kamphuis C, Barsom E, Schijven M, Christoph N. Augmented reality in medical education? Perspect Med Educ. 2014;3(4):300-311.

10. Ziv A, Wolpe PR, Small SD, Glick S. Simulation-based medical education: an ethical imperative. Simul Healthc. 2006;1(4):252-256.

11. Donaldson MS, Corrigan JM, Kohn LT. To Err Is Human: Building a Safer Health System. Vol. 6. Washington, DC: National Academies Press; 2000.

12. Barsuk JH, Cohen ER, Mikolajczak A, Seburn S, Slade M, Wayne DB. Simulation-based mastery learning improves central line maintenance skills of ICU nurses. J Nurs Adm. 2015;45(10):511-517.

13. Eggimann P, Harbarth S, Constantin M-N, Touveneau S, Chevrolet J-C, Pittet D. Impact of a prevention strategy targeted at vascular-access care on incidence of infections acquired in intensive care. Lancet. 2000;355(9218):1864-1868.

14. Barsuk JH, Cohen ER, Feinglass J, McGaghie WC, Wayne DB. Use of simulation-based education to reduce catheter-related bloodstream infections. Arch Intern Med. 2009;169(15):1420-1423.

15. Jagneaux T, Caffery TS, Musso MW, et al. Simulation-based education enhances patient safety behaviors during central venous catheter placement. J Patient Saf. Epub 2017 Oct 4.

16. Centers for Medicare and Medicaid Services (CMS), HHS. Medicare program: changes to the hospital inpatient prospective payment systems and fiscal year 2009 rates; payments for graduate medical education in certain emergency situations; changes to disclosure of physician ownership in hospitals and physician self-referral rules; updates to the longterm care prospective payment system; updates to certain IPPS-excluded hospitals; and collection of information regarding financial relationships between hospitals. Final rules. Fed Regist. 2008;73(161):48433-49084.

17. Klopfer E, Sheldon J. Augmenting your own reality: student authoring of science-based augmented reality games. New Dir Youth Dev. 2010;2010(128):85-94.

18. Hugues O, Fuchs P, Nannipieri O. New augmented reality taxonomy: technologies and features of augmented environment. Handbook of Augmented Reality. Furht B, editor. New York: Springer-Verlag; 2011:47-63.
19. Johnson LF, Levine A, Smith RS, Haywood K. Key emerging technologies for elementary and secondary education. Educ Dig. 2010;76(1):36.

20. Johnson LF, Levine A, Smith RS, Haywood K. Key emerging technologies for postsecondary education. Educ Dig. 2010;76(2):34.

21. Jacobus CJ, Griffin JL. Method and system for simulating medical procedures including virtual reality and control method and system for use therein. Google Patents US5769640A. 1998 Jun 23.

22. Fuchs H, Livingston MA, Raskar R, et al. Augmented reality visualization for laparoscopic surgery. Paper presented at: International Conference on Medical Image Computing and Computer-Assisted Intervention; October 11-13, 1998; Cambridge, MA, USA.

23. Marescaux J, Rubino F, Arenas M, Mutter D, Soler L. Augmented-realityassisted laparoscopic adrenalectomy. JAMA. 2004;292(18):2211-2215.

24. Geiger B, Kikinis R. Simulation of endoscopy. Lect Notes Comput Sci. 1995;905:277-281.

25. The Joint Commission [webpage on the Internet]. CLASBI Prevention Strategies, Techniques, and Technologies. 2017. Available from: https:// www.jointcommission.org/topics/clabsi_toolkit_chapter_3.aspx. Accessed October 30, 2017.

26. Ortega R, Song M, Hansen CJ, Barash P. Videos in clinical medicine. Ultrasound-guided internal jugular vein cannulation. $N$ Engl J Med. 2010;362(16):e57.

27. Santos ME, Polvi J, Taketomi T, Yamamoto G, Sandor C, Kato H. Toward standard usability questionnaires for handheld augmented reality. IEEE Comput Graph Appl. 2015;35(5):66-75.

28. Tehrani DM, Russell D, Brown J, et al. Discord among performance measures for central line-associated bloodstream infection. Infect Control Hosp Epidemiol. 2013;34(2):176-183.

29. Sznajder JI, Zveibil FR, Bitterman H, Weiner P, Bursztein S. Central vein catheterization. Failure and complication rates by three percutaneous approaches. Arch Intern Med. 1986;146(2):259-261.

30. Philibert I, Barach P. Residents' hours of work. BMJ. 2002;325(7374): 1184-1185.

31. Philibert I, Friedmann P, Williams WT. Education AWGoRDHACfGM. New requirements for resident duty hours. JAMA. 2002;288(9):1112-1114.

32. Krummel TM. Surgical simulation and virtual reality: the coming revolution. Ann Surg. 1998;228(5):635-637.

33. Reznek M, Harter P, Krummel T. Virtual reality and simulation: training the future emergency physician. Acad Emerg Med. 2002;9(1):78-87.

34. Reznek MA, Rawn CL, Krummel TM. Evaluation of the educational effectiveness of a virtual reality intravenous insertion simulator. Acad Emerg Med. 2002;9(11):1319-1325.

35. McGee DC, Gould MK. Preventing complications of central venous catheterization. $N$ Engl J Med. 2003;348(12):1123-1133.

36. Wu H-K, Lee SW-Y, Chang H-Y, Liang J-C. Current status, opportunities and challenges of augmented reality in education. Comput Educ. 2013;62:41-49.

37. Dunleavy M, Dede C, Mitchell R. Affordances and limitations of immersive participatory augmented reality simulations for teaching and learning. J Sci Educ Technol. 2009;18(1):7-22.

38. Akçayır M, Akçayır G. Advantages and challenges associated with augmented reality for education: a systematic review of the literature. Educ Res Rev. 2017;20(Suppl C):1-11.
Advances in Medical Education and Practice

\section{Publish your work in this journal}

Advances in Medical Education and Practice is an international, peerreviewed, open access journal that aims to present and publish research on Medical Education covering medical, dental, nursing and allied health care professional education. The journal covers undergraduate education, postgraduate training and continuing medical education

\section{Dovepress}

including emerging trends and innovative models linking education, research, and health care services. The manuscript management system is completely online and includes a very quick and fair peer-review system. Visit http://www.dovepress.com/testimonials.php to read real quotes from published authors. 